\title{
Der ventrale Zuggurtungsfixateur zur Behandlung der proximalen Tibiapseudarthrose
}

$\square$ Klaus Michael Stürmer

Die 20-jährige Patientin Yvonne M. erleidet als Beifahrerin einen Motorradunfall und zieht sich dabei eine erstgradig offene Unterschenkel-Stückfraktur links zu (Abb.1). Die Unterschenkelfraktur wird primär versorgt: Weichteildébridement und gedeckte Marknagelung (ACE, DePuy) ohne Aufbohren der Markhöhle. Die Verriegelung erfolgt zuerst distal, um danach den Nagel noch etwas zurückzuschlagen und so die Fragmente besser ineinanderstauchen zu können; jetzt erst folgt die proximale Verriegelung. Die intraoperative Durchleuchtung zeigt, dass der ventrale proximale Frakturspalt tendenziell etwas klafft. Da dies erfahrungsgemäß an der proximalen Tibia zu Heilungsstörungen führen kann, entschließt sich der Operateur zur zusätzlichen Montage einer 3,5-mm-Kleinfragmentplatte (Synthes) an der ventralen Tibiakante mit dem Ziel, hier einen Zuggurtungseffekt zu erreichen. Die Reposition gelingt einwandfrei auf beiden Etagen (Abb. 2).

Die Weichteile heilen problemlos und ohne jeden Hinweis auf Infekt ab. Die Patientin wird nach 1 Woche an Unterarmgehstützen gehend entlassen. Sie rollt den linken Fuß die ersten 4 Wochen mit 10-20 kg Belastung ab. Danach steigert sie die Belastung bis zur Schmerzgrenze und kann nach 3 Monaten ohne Gehstützen gehen. Die distale Fraktur ist zu diesem Zeitpunkt radiologisch und klinisch verheilt, die proximale Fraktur ist noch nicht vollständig durchbaut. Es bestehen proximal belastungsabhängige Schmerzen, die aber erträglich sind.

Nach 12 Monaten ist die proximale Fraktur immer noch nicht fest, die Platte ist locker und die Fraktur klafft ventral (Abb. 3). Die Schmerzen bei Belastung

OP-JOURNAL 2008; 24: 244-245

(c) Georg Thieme Verlag KG Stuttgart • New York DOI 10.1055/s-2008-1039125

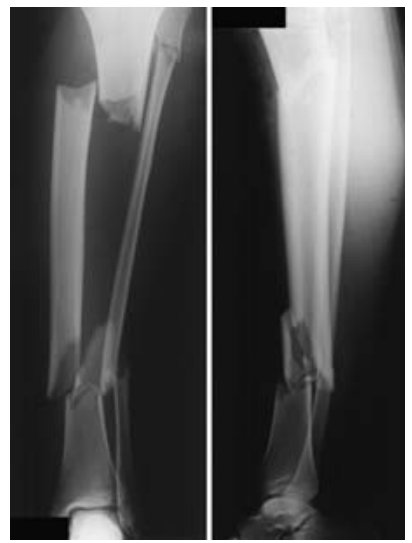

Abb. 1 Unterschenkelstückfraktur, 1-gradig offen.

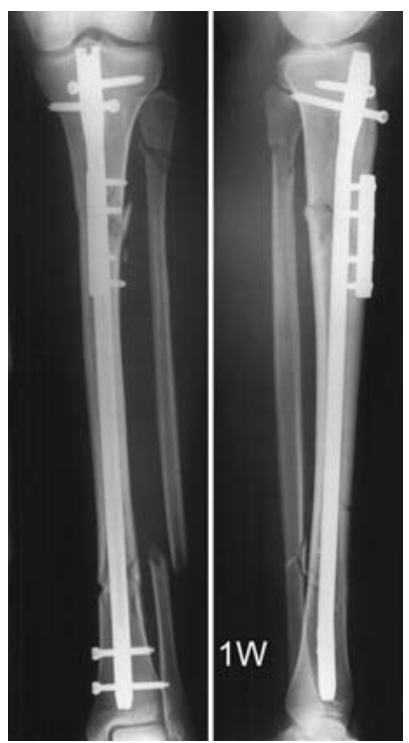

Abb. 2 Marknagel unaufgebohrt mit ventraler Zuggurtungsplatte, 1 Woche.

haben zugenommen. Daraufhin wird die Platte entfernt und der Marknagel distal dynamisiert. Nach 18 Monaten ist die Fraktur immer noch nicht durchbaut und die Schmerzen nehmen zu (Abb.4). Daraufhin wird auch proximal entriegelt, jedoch wieder ohne Erfolg: Die Pseudarthrose besteht klinisch und

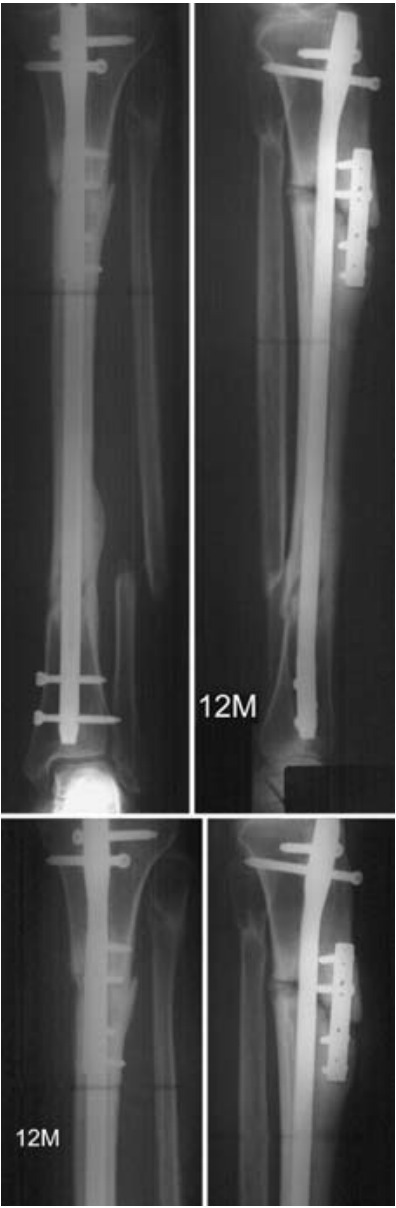

Abb. 3 Pseudarthrose der proximalen Fraktur, 12 Monate.

radiologisch nach 24 Monaten weiter (Abb. 5).

Jetzt wird ein ventraler Fixateur externe montiert, der über eine Gewindespindel stark vorgespannt wird (Abb. 6). Dieser Fixateur funktioniert als ventrale Zuggurtung und neutralisiert biomechanisch die ventral über die Patellarsehne und dorsal über den $\mathrm{M}$. gastrocnemius wirkenden Kräfte am Tibiakopf. Bereits einen Monat später ist die Patientin absolut beschwerdefrei und die gut vaskularisierte Pseudarthrose baut auch 


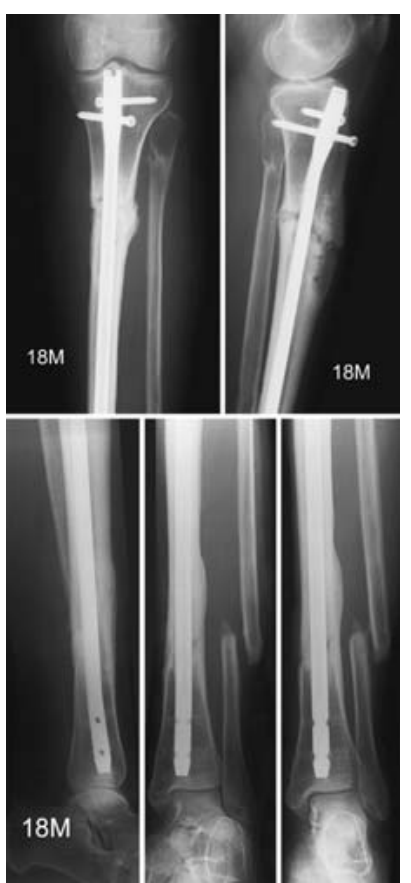

Abb. 4 Persistierende Pseudarthrose trotz distaler Dynamisierung und Plattenentferung, 18 Monate.

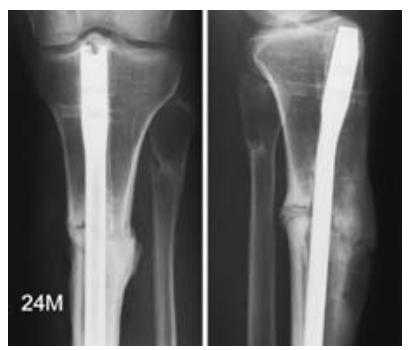

Abb. 5 Proximale Dynamisierung: persistierende Pseudarthrose, 24 Monate.

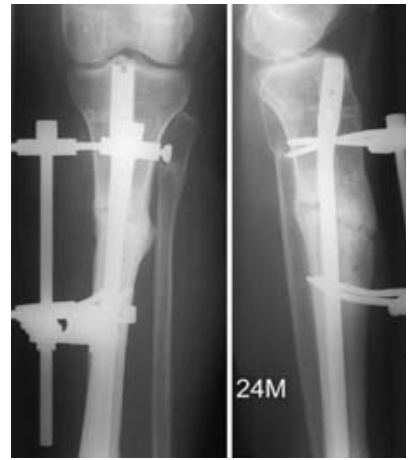

Abb. 6 Ventraler Zuggurtungsfixateur, stark vorgespannt, 24 Monate.

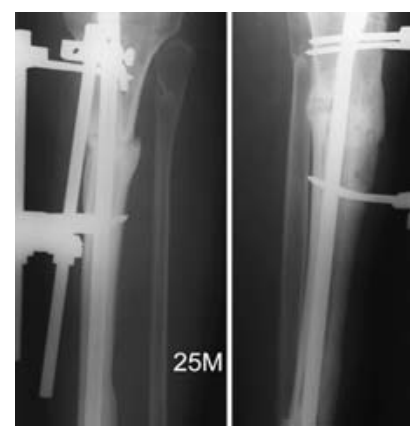

Abb. 7 Durchbau der Pseudarthrose einen Monat später, 25 Monate.

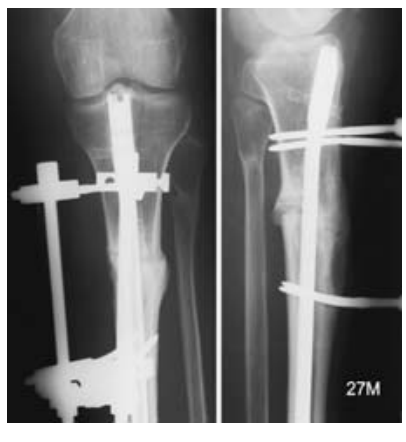

Abb. 8 Geheilte Pseudarthrose nach 27 Monaten.

effektiven ventralen Zuggurtung, entweder durch eine Zuggurtungsplatte oder noch besser durch einen hier gezeigten ventral vorgespannten Fixateur externe. Die Pseudarthrose baut dann sehr schnell spontan durch und die belastungsabhängigen Schmerzen sind in der Regel sofort weg. Wir überblicken bisher 9 derartige Fälle, die unter dieser Therapie erfolgreich zur Heilung gebracht werden konnten. Von einer Resektion der Pseudarthrose oder Spongiosaplastik raten wir ab.

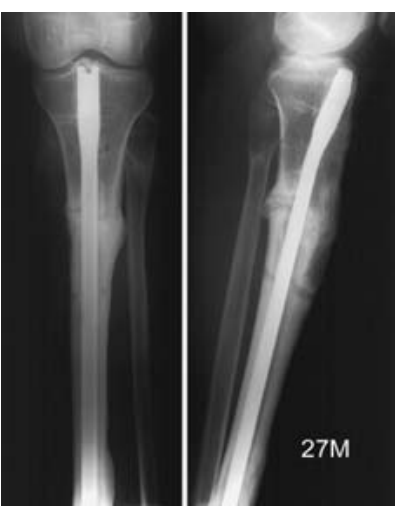

Abb. 9 Geheilte Paseudarthrose nach Entferung des Fixateur externe.

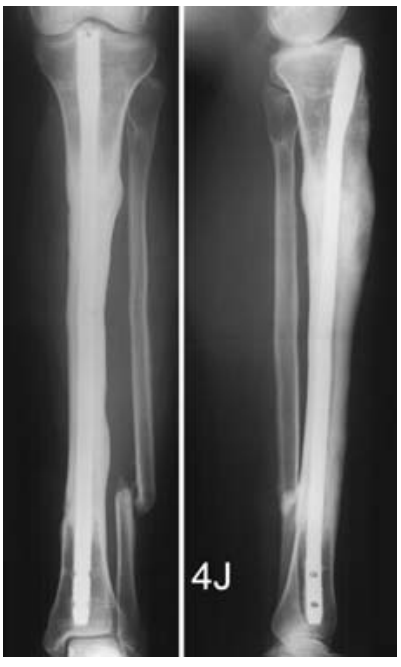

Abb. 10 Langzeitergebnis 4 Jahre nach dem Unfall.

\section{Prof. Dr. Klaus Michael Stürmer} Direktor

Klinik für Unfallchirurgie, Plastische und Wiederherstellungschirurgie Universitätsklinikum der Georg-August-Universität Göttingen Robert-Koch-Straße 40 37075 Göttingen

E-Mail: ms.unfallchirurgie@ med.uni-goettingen.de

Proximale Tibiapseudarthrosen sind in der Regel vom hypertrophen, gut vaskularisierten Typ. Es handelt sich um ein rein biomechanisches Problem. Die optimale Therapie besteht in einer 